\section{Dual-Band Switched-Beam Antenna Using Slot Active Frequency Selective Surface}

\author{
Chao Gu, B. Sanz-Izquierdo, Steven Gao, J. C. Batchelor, \\ E. A. Parker, Fan Qin, Gao Wei, Jianzhou Li and Jiadong \\ $\mathrm{Xu}$
}

\begin{abstract}
A dual-band electronically beam-switching antenna using a cylindrical active frequency selective surface (AFSS) is presented. The antenna structure consists of a slot AFSS and an omnidirectional dual-band feed antenna. The PIN diodes of half the AFSS are switched ON and OFF respectively to reconfigure the surface as transparent and opaque to the incident EM waves. Thus by controlling the bias voltages to different columns, the directive beams can be swept in 4 steps covering the whole azimuth plane. A design validation based on a metallic reflector antenna is performed to predict the performances of the proposed AFSS antenna. To validate the design concept, the transmission coefficients of the active FSS sheet are experimentally examined. Then an antenna prototype operating at $2.5 \mathrm{GHz}$ and $5.3 \mathrm{GHz}$ frequency bands is fabricated and measured. Simulation and measurement results of the fabricated antenna are presented to verify the design principle. A comparison between this work and previous (single-band) designs is given to better interpret the characteristics of the dual-band AFSS antenna.
\end{abstract}

Index Terms -Dual-band antennas, frequency selective surface, reconfigurable antennas, electronically beam-steering antennas.

\section{INTRODUCTION}

IN the last two decades, wireless communication systems have become one of the fastest growing and most dynamic sectors in the communication industry. New versatile antennas which can satisfy the increasing demand are expected to adapt their performances to the new system requirements. Reconfigurable antennas (RAs) and smart antennas are good candidates to deliver the performance necessary to support emerging applications and future wireless systems. However, conventional smart antennas e.g. phased arrays need to integrate phase shifters, power splitters and control systems, which make them high-cost, bulky and low efficient. To overcome this problem, research effort should be spent on RAs that mitigate the aforementioned detriments to conventional smart antennas.

Beam-switching RAs can be used in mobile and indoor communication systems to improve wireless link quality and enhance spatial reuse. There are various techniques for beamswitching antennas in the literature [1]-[9]. Most of them operate at a single frequency band. In [6], [7], reactive loads e.g. PIN diodes and varactors are used to steer the antenna main beam in the horizontal plane, which eliminates the need for phase shifters. RAs presented in [10]-[12] are capable of dual-

Manuscript received October 9, 2001. (Write the date on which you submitted your paper for review.) This work was supported in part by the U.S. Department of Commerce under Grant BS123456 (sponsor and financial support acknowledgment goes here). band beam-switching operation. However, the steered beams cannot cover the entire horizontal plane. Recently, with the proliferation of artificial materials, a new class of beamswitching antennas incorporating reconfigurable frequency selective surfaces (FSSs) have received increasing research interest [13]-[22]. Known as filters of electromagnetic waves, FSSs can transmit or reflect the incident wave according to frequency. They are commonly accomplished by etching periodic patterns on metallized dielectric materials, which is practical for integration with antennas. By embedding active control devices into FSSs, switching and tuning of the transmission response can be achieved. The proposed antennas in [15] are composed of an omnidirectional antenna source surrounded by a reconfigurable cylindrical FSS structure which is divided into several sectors. By switching the PIN diodes in the specific sectors of the AFSS, one sector can be treated as almost transparent and the others are opaque at the desired frequencies. Mimicking a perfect electric conductor backing a dipole antenna, the AFSS is able to convert the omnidirectional beam into a directional one at the chosen angles. Therefore, the beam can be steered over the entire horizontal plane by means of electronically adjusting the states of all sectors.

In this paper, a dual-band electronically beam-switching antenna is proposed for the first time by utilizing a cylindrical AFSS. A dual-band dipole antenna is carefully designed to illuminate the AFSS. Enclosing the radiation source, the cylindrical FSS is composed of ten columns each with twelve unit cells. The design features in [23] are exploited to develop a new dual-band unit cell using square loops. The slot-form square loop elements are etched on one side of a thin substrate with the biasing circuit on the other side, which eliminates the use of metallic vias. The cylindrical AFSS can be divided into two sections where the PIN diodes have different ON/OFF states. One FSS section acts as a reflector when the PIN diodes on it are all switched ON, whereas the other section works as a transparent EM window at the resonant frequency. Consequently, similar to the working principle of corner reflector antennas, a directional beam can be generated and swept over the whole azimuth plane due to the antenna structure symmetry. In this design, each dual-band unit cell incorporates four PIN diodes with a series biasing circuit.

The rest of this paper is organized as follows. In Section II, the detailed design of the unit cell is presented together with studies of the effect of design parameters on the transmission response. In Section III, the operation principle of the presented antenna is described including the dual-band feed antenna design and beam-switching mechanism. In addition, a brief design guideline based on a metallic semi-cylindrical reflector is also introduced to predict the AFSS antenna performance. Simulation and experimental results are presented and compared in Section IV. Finally, conclusions are drawn in

C. Gu, B. Sanz-Izquierdo, S. Gao, J. C. Batchelor, E. A. Parker are with School of Engineering and Digital Arts, University of Kent, Canterbury, CT2 7NT, UK (e-mail: cg342@kent.ac.uk).

T. C. Author is with the Electrical Engineering Department, University of Colorado, Boulder, CO 80309 USA, on leave from the National Research Institute for Metals, Tsukuba, Japan (e-mail: author@ nrim.go.jp). 
Section V.

\section{ACtive FSS UNIT CELL DeSIGN}

The key to realizing a FSS based beam-switching antenna is to switch the FSS between transmitting and reflecting modes using PIN diodes. To that end, a reconfigurable dual-band FSS unit cell should be designed. As a good candidate, active slotelement FSSs have been reported in [24], where a switchable double-sided structure is employed. The passive slot FSS elements are etched on one side of the thin substrate with the biasing circuit connecting PIN diodes in series on the other side. Also, the switching properties of AFSS based on two concentric split ring resonators were proposed in [25]. Here we propose a new dual-band AFSS structure, where a dual band-pass response can be obtained by tuning the two concentric square slot rings shown in Fig. 1. The proposed square unit cell and its transmission coefficients were simulated based on Floquet theorem. In this configuration, the EM plane waves are impinging upon the FSS with the E field parallel to the $\mathrm{y}$ direction. The lower resonant frequency is caused by the outer loop and the higher resonant frequency is dictated by the inner loop. By incorporating PIN diodes into the unit cell, the two concentric loops are split, which shifts the two resonant frequencies. Indeed, switching the PIN diodes on or off changes the effective inductance and capacitance of the loop slots. The FSS elements were etched on $0.05 \mathrm{~mm}$ thick flexible substrate with $\varepsilon_{\mathrm{r}}=2.8$. This thin substrate is used to form the planar FSS into a cylindrical one. As can be seen from Fig. 1(b), the four PIN diodes are placed at different positions for each slot. When these diodes are deactivated or activated, in the ideal case, the sheet can be reconfigured as transparent or opaque at the resonant frequencies. The BAR 64-02V silicon PIN diode was selected and modeled as a typical forward resistance $R s=2.1 \Omega$ $(0.85 \Omega \leq R s \leq 2.8 \Omega)$ for the $\mathrm{ON}$-state and a parallel connection of a capacitance $C_{s}=0.17 \mathrm{pF}$ in the OFF state. To obtain the required dual-band pass responses, the related design parameters of the element geometry should be optimize and the final dimensions of the resulting AFSS unit cell are given in Table I. Note that the periodicity p of the square unit cell applies for both $\mathrm{x}$ and $\mathrm{y}$ directions.

The infinite passive and active FSS were simulated and the computed magnitude of transmission coefficients are shown in Fig. 2. Three kinds of configurations were examined with the same geometry dimensions. It can be seen clearly that by loading the four OFF-state PIN diodes, the pass-band of the passive FSS is down-shifted significantly due to the added capacitance between the gaps of the two slots. To study the effect of the bias circuit (BC), the feed lines were removed whereas diodes were kept between the gaps of passive element. It is observed that the $\mathrm{BC}$ has only a minor effect on the transmission responses. At normal incidence, the presented AFSS resonates at $2.5 \mathrm{GHz}$ and $5.5 \mathrm{GHz}$ when all diodes are OFF, whereas a transmission level below $-15 \mathrm{~dB}$ between 2 to $6 \mathrm{GHz}$ is achieved with the diodes in the on-state. The reflection coefficient levels found in the on / off state for the two bands were less than $0.3 \mathrm{~dB}$ and $-15 \mathrm{~dB}$, respectively.
Although $a, b$, and $l$ are the three main parameters to tune the unit cell, the electrical characteristics of the PIN diodes cannot be neglected. It is noted that $R s$ almost has no impact on the magnitude of the transmission coefficient whereas a small change of diode capacitance leads to significant shift of the passband location. The frequency response at $2.5 \mathrm{GHz}$ is relatively stable when changing the bias line width. The very thin substrate $(0.05 \mathrm{~mm})$ were found to have almost no effect on the AFSS transmission responses.

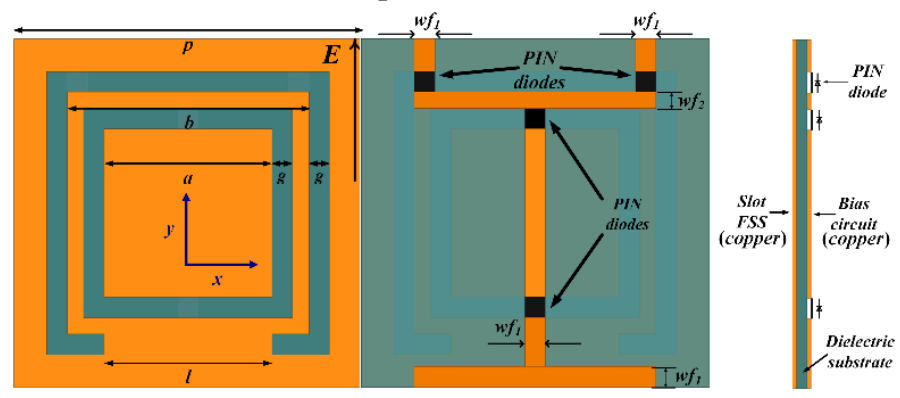

Fig. 1. AFSS unit cell configurations: (a) front view with two concentric slots, (b) back view with bias circuit, (c) side view of the biasing scheme.

(a)

TABLE I DIMENSIONS OF UNIT CELL GEOMETRY [mm] (c)

\begin{tabular}{ccccccc}
\multicolumn{8}{c}{ DIMENSIONS OF } & UNIT CELL GEOMETRY $[\mathrm{mm}]$ & (c) \\
\hline$p$ & $b$ & $g$ & $a$ & $l$ & $w f 1$ & $w f 2$ \\
\hline 17 & 11.8 & 1 & 8.2 & 8.2 & 1 & 0.8 \\
\hline
\end{tabular}

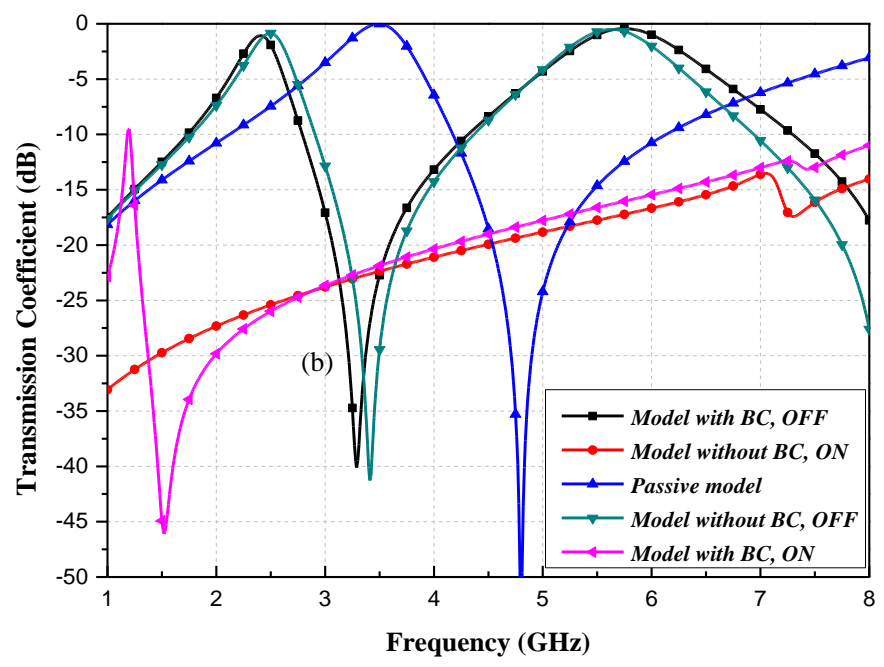

Fig. 2. Simulated magnitude of the AFSS transmission coefficients.

\section{ANTENNA DESIGN PRINCIPLE}

\section{A. Antenna Structure and Operation Principle}

Fig. 3 shows the schematic of the proposed antenna. As can be seen form the figure, the antenna is composed of a reconfigurable cylindrical FSS enclosing a dual-band dipole. The FSS structure consists of unit cells described in Section II. Ten columns of AFSS are employed and placed cylindrically with an angular periodicity of $\theta_{f s s}=36^{\circ}$ and a radius of $R_{f s s}=27$ $\mathrm{mm}$. Each column has twelve unit cells and the total length of the antenna $L_{f s s}$ is $204 \mathrm{~mm}$. For clarity, some columns are made transparent. Substrate and bias lines are not shown in Fig. 3. 

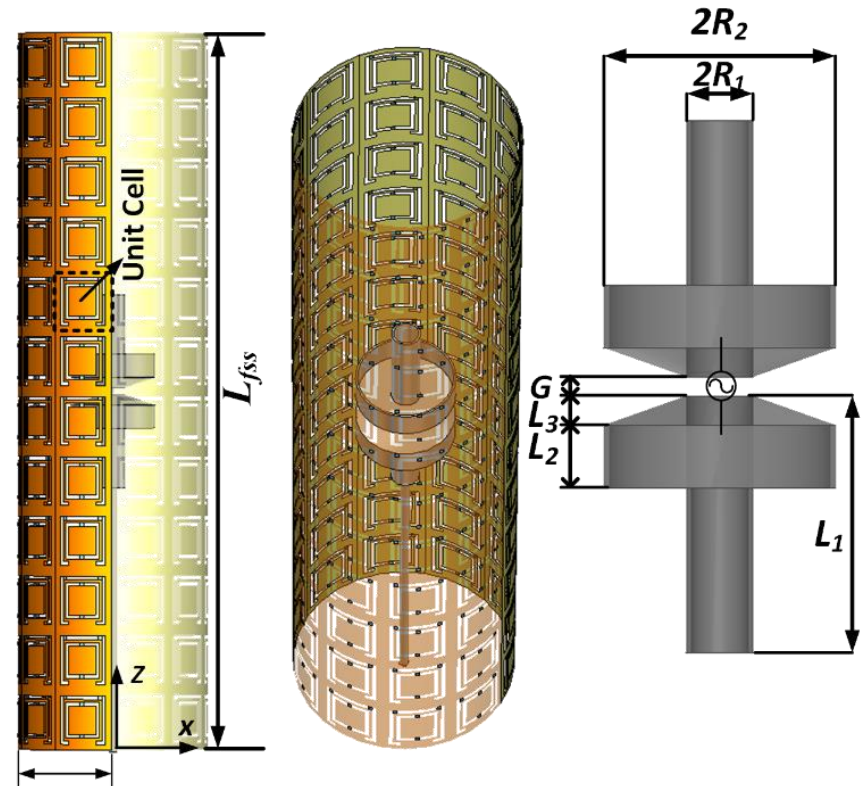

$R_{f s s}$

Fig. 3. Antenna (a) side view, (b) perspective view.

The dual-band dipole feed antenna is placed along the axis at the center of the cylindrical FSS. To transform the omnidirectional pattern into a directional one, 10 columns (parallel to the cylinder axis) are divided into 2 sectors. In this work, 4 beams are generated to cover the horizontal (x-y) plane in $90^{\circ}$ steps. In each step, the diodes of one sector are OFF while those in the other are ON. According to the simulated results in Section II, the semi-cylinder sector with ON-state diodes reflects the incident EM wave while the sector with OFF-state diodes is transparent. By switching the diodes of each sector, the antenna radiation pattern can be swept in four steps with the same bias configurations due to the structure symmetry.

TABLE II

DIMENSIONS OF ANTENNA GEOMETRY [mm]

\begin{tabular}{|c|c|c|c|}
\hline$R f s s$ & $L f s s$ & $R 1$ & $R 2$ \\
\hline 27 & 204 & 3.5 & 12 \\
\hline$G$ & $L 1$ & $L 2$ & $L 3$ \\
\hline 2 & 26.5 & 3 & 6.5 \\
\hline
\end{tabular}

\section{B. AFSS Antenna Design}

The radiation characteristics of the metallic reflector antenna are carefully examined to predict the proposed AFSS antenna. When fabricating, the planar AFSS is conformed to a cylinder whose radius is equal to that of the simulated metallic reflector. Based on the designed unit cell periodicity $P$, we can calculate the number of the unit cells along the circle circumference using $N=2 \times \pi \times R / P$. Also, the unit-cell number along the reflector length can be calculated by $M=L / P$. So here it is expected that with $\mathrm{N}=10$ and $\mathrm{M}=12$ the presented AFSS antenna can have comparable performances as the validated dual-band semi-cylindrical reflector antenna. For all the four antenna beam-switching cases, 5 of 10 columns of PIN diodes need to be forward biased. According to the measurement setup, switching on each of the five parallel columns requires $35 \mathrm{~V}$ DC power supply. Then the DC current flowing in each column is $20 \mathrm{~mA}$. Thus, the total power consumption for each case is calculated by $\mathrm{P}=35 \times(0.02 \times 5)=3.5 \mathrm{w}$. The AFSS antenna structure was constructed and simulated in commercial fullwave EM software CST. To avoid generating large numbers of mesh-cells, the bias lines were removed when simulating.

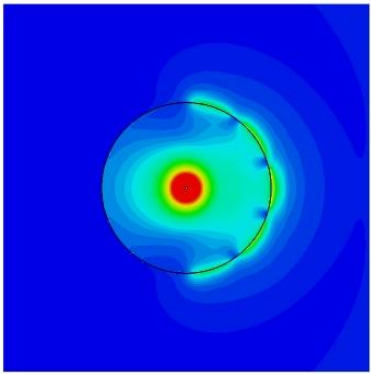

(a)

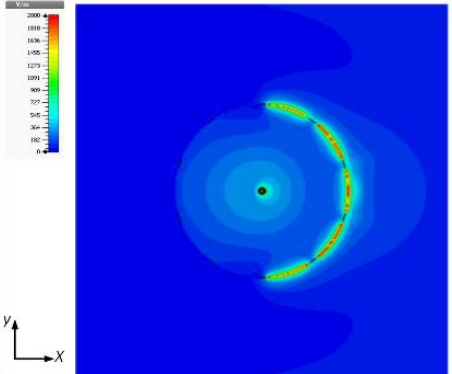

(b)
Fig. 4. Simulated E-field distribution at (a): $2.5 \mathrm{GHz}$ and (b): $5.3 \mathrm{GHz}$.

Fig. 4 shows the simulated E-field distribution of the AFSS antenna at $2.5 \mathrm{GHz}$ and $5.3 \mathrm{GHz}$. It can be seen that the E-field of the feed antenna is reflected into one direction at both the two frequencies, which agrees well with the design principle. Fig. 5 presents the simulated radiation patterns at $2.5 \mathrm{GHz}$ and 5.3GHz. Compared with results for a metallic reflector antenna, it is noted that the side-lobe and back-lobe levels of the AFSS antenna are deteriorated because the reconfigurable FSS is not ideally reflective or transparent.

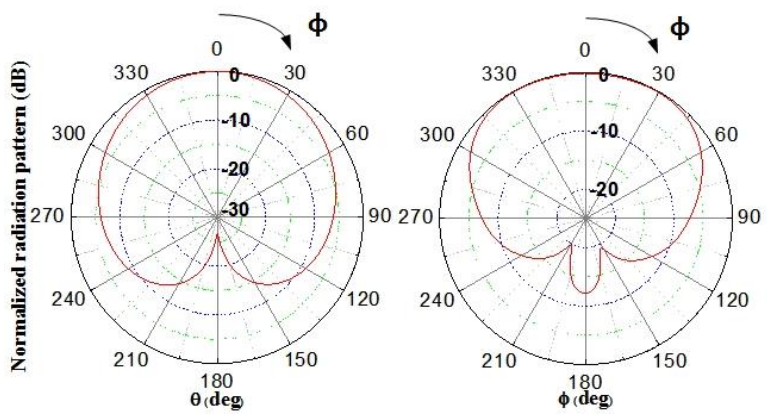

(a)
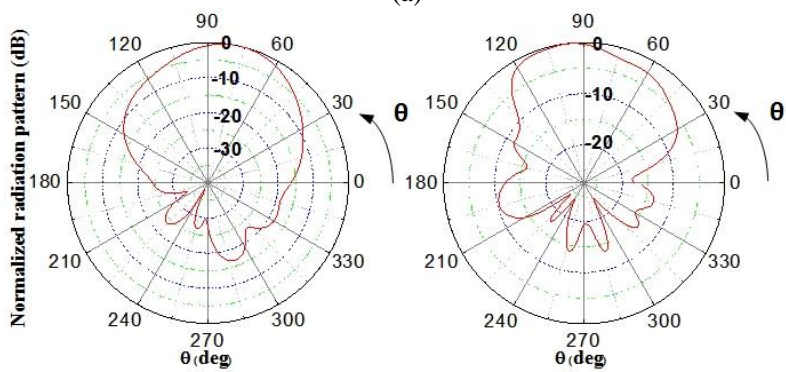

(b)

Fig. 5. Simulated radiation patterns at $2.5 \mathrm{GHz}$ and $5.3 \mathrm{GHz}$ (a) H-plane, (b) E-plane.

\section{EXPERIMENTAL RESULTS AND DISCUSSION}

To validate the presented operation principle, an antenna prototype was fabricated and measured. First, the performance of the planar AFSS in Section II was tested by measuring the transmission coefficients. The planar FSS consisted of $10 \times 12$ unit cells of the dimensions in Fig.1 and Table I. A schematic of the full biasing circuit is shown in Fig.6 (a) and a detailed photograph in Fig.6 (b). Ten parallel bias lines were etched, and RF chokes (PANASONIC ELJQE3N9ZFA) were added at one 
of their ends. At the other end, the lines connected together to a common line that was connected to the ground which consists of the slot FSS copper layer. $1 \mathrm{~K} \Omega$ resistor was added between in order to limit the current to the diodes.

Two broadband antennas connected to a network analyzer were used in a test chamber. Fig. 7 compares the measured and simulated transmission coefficients of the AFSS when all the diodes are switched on / off. When the diodes were in the offstate, a parallel resistance $\left(\mathrm{R}_{\mathrm{P}}\right)$ was added into the simulation to obtain more accurate results. It can be noted that the frequency responses of the two states agree well with results in Section II except that the discrepancy is approximately $1 \mathrm{~dB}$ for the higher band. This is assessed to be caused by errors in the measurement set up, as well as the diode package and substrate losses. The measurement errors also relate to the small array size and small isolation screen aperture used for the tests as well as the nonplane wave characteristics of the transmitted signal.

The fabricated cylindrical prototype is shown in Fig. 8. When measuring the $S_{11}$ magnitude, half of the AFSS columns were activated. Theoretically, due to the antenna geometrical symmetry, the measured $\left|S_{11}\right|$ is expected to be the same by randomly switching on five neighboring columns. Thus the antenna performances were evaluated with a randomly selected beam direction.

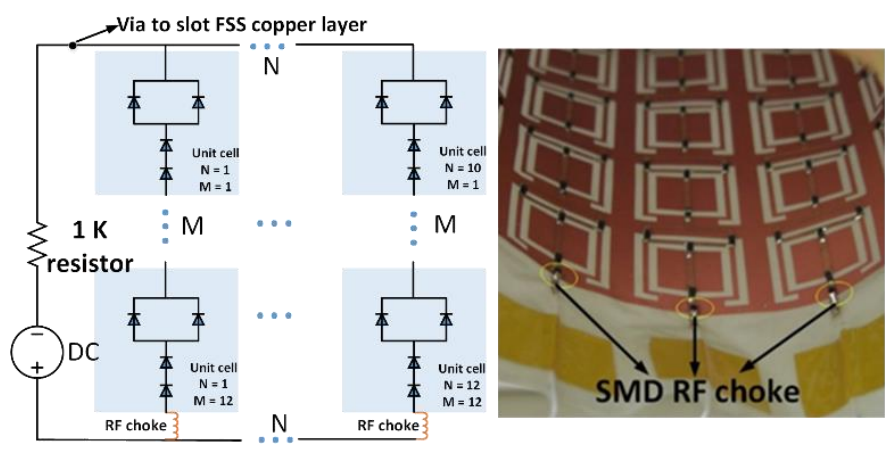

Fig. 6. Schematic of the AFSS biasing network.

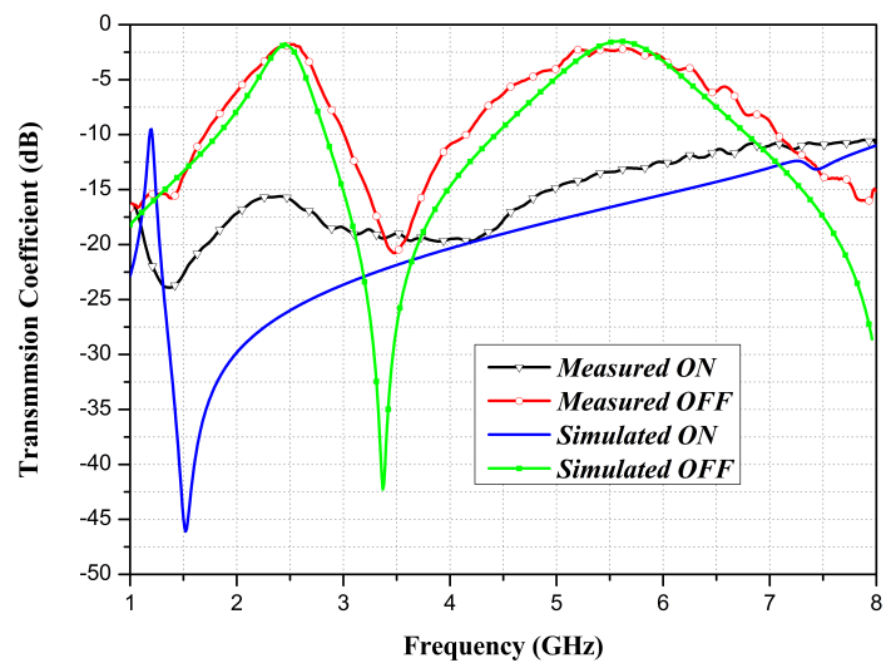

Fig. 7. Measured transmission coefficients when diodes are OFF/ON.

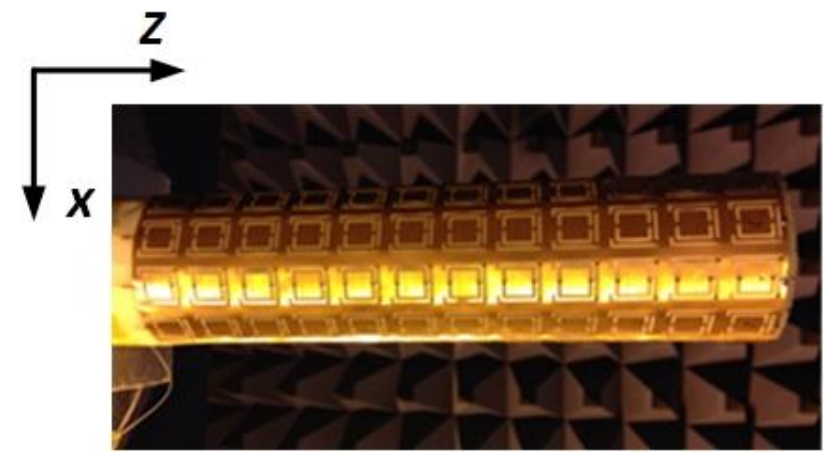

Fig. 8. Photograph of the fabricated antenna

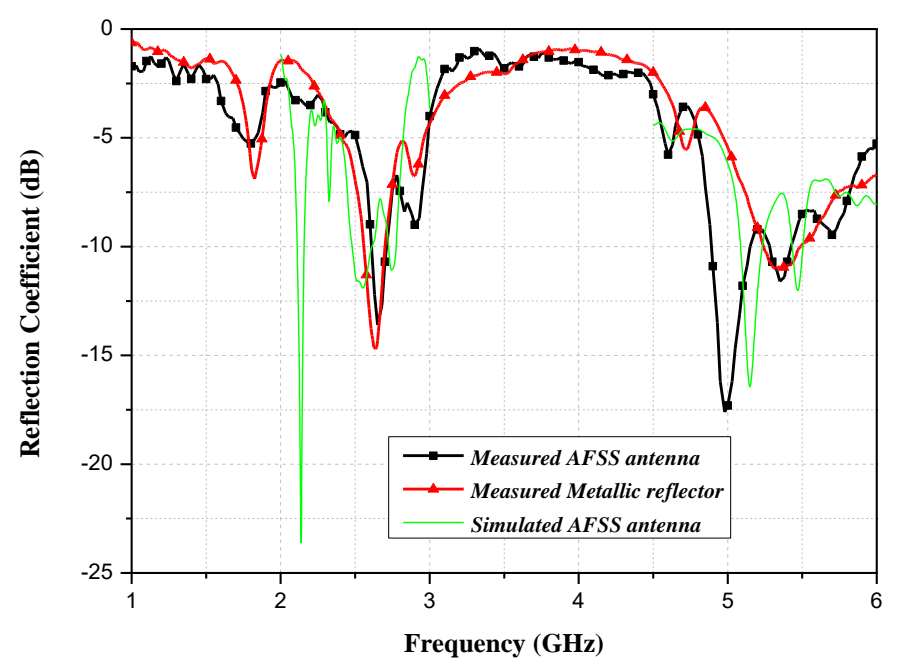

Fig. 9. Measured reflection coefficients of the fabricated antennas

For the measurements, five adjacent columns of diodes were $\mathrm{OFF}$ and the others were ON. The OFF state requires a DC voltage of $0 \mathrm{~V}$ and $35 \mathrm{~V}$ DC power supply was applied to the five parallel bias lines to switch on the didoes. For comparison, the measured $\left|S_{11}\right|$ of the metallic reflector antenna is also presented. Fig. 9 compares the simulated and measured $\left|S_{11}\right|$ results of the AFSS antenna and the reference antenna. The impedance matching of the proposed AFSS antenna is different from the reference antenna at the higher band and both the higher resonant frequencies shift downwards compared to the simulation results in Section III.

The measured radiation patterns of the presented antenna are shown in Figs. $10 \&$ 11. The $3 \mathrm{~dB}$ H-plane beamwidths of the two operation bands are $90^{\circ}$ and $100^{\circ}$, respectively. The highest back-lobe level at lower frequencies is less than $-18 \mathrm{~dB}$ while it is $-12 \mathrm{~dB}$ at the higher frequencies. The normalized E-plane patterns show less than $-12 \mathrm{~dB}$ side-lobe level at higher band whereas a side-lobe of $-12 \mathrm{~dB}$ is the best at $2.5 \mathrm{GHz}$ among the three measured results. Moreover, the cross-polarization levels across the $3 \mathrm{~dB}$ beamwidths of the two planes are below $-12 \mathrm{~dB}$. It can be noticed that the main beams of the higher band have $20^{\circ}$ beam tilt from the horizontal plane, which is caused by placing the coaxial feed cable asymmetrically in the cylindrical AFSS. The length of the cable is more than one wavelength at $5 \mathrm{GHz}$, which deteriorates the antenna beam at the higher operation band. This distortion could be reduced using 
absorbing material. However, this may lead to a reduction in antenna radiation efficiency. Another possibility is the use of a differentially fed dual-band dipole as the feeder or a dual-band sleeve balun.

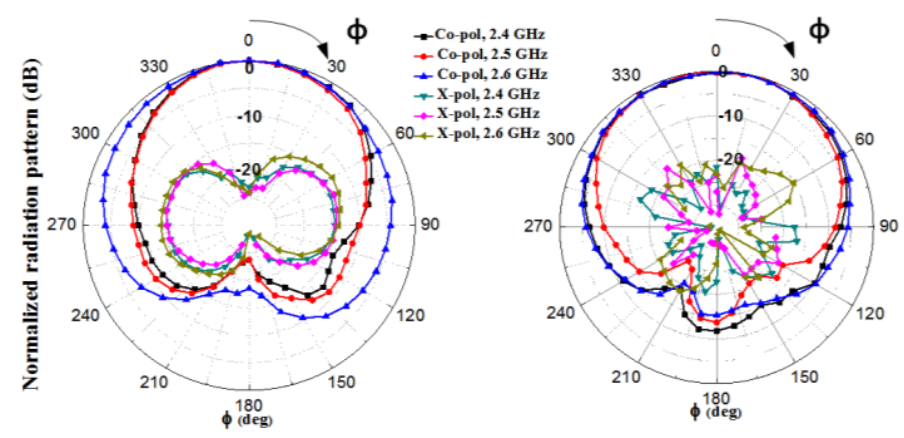

Fig. 10. Measured H-plane radiation patterns.

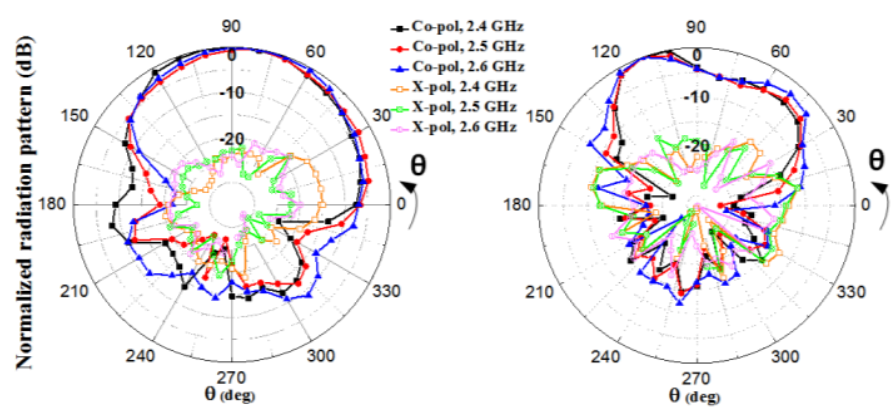

Fig. 11. Measured E-plane radiation patterns.

Fig. 12 shows the measured gains of the AFSS antenna when the four beams are generated in the horizontal plane. The gain was measured at the cut plane of the maximum radiation. As can be observed, the proposed antenna offers gains of $6.5 \mathrm{dBi}$ and $5 \mathrm{dBi}$ at the two desired frequencies. Moreover, it is concluded that the radiation patterns can be switched in 4 steps to cover the whole azimuth plane at both frequencies. Compared with the simulated results, the realized gains are reduced mainly due to the considerable transmission loss (e.g. $2.5 \mathrm{~dB}$ at $5.3 \mathrm{GHz}$ ) since the EM wave energy is notably degraded when the AFSS is switched on as an EM transmitting window. Fig. 13 shows the antenna efficiency at the two frequency bands. It appears that there are degradations for both the two bands especially the higher one. The reason for that is the low realized gain compared with the simulated one. More specifically, the loss of the PIN diodes at $5 \mathrm{GHz}$ is increased significantly. Also, the loss of the thin substrate attributes to the problem as we didn't include it in the simulation. This issue could be mitigated by low-loss RF switches, e.g. MEMS. However employing MEMS switches will lead to a high control voltage typically larger than $70 \mathrm{~V}$ given that the series bias circuit in the AFSS design. On the other hand, most off-theshelf low-loss PIN diodes (e.g. MACOM MA4SPS42X) operating larger than $5 \mathrm{GHz}$ have smaller package size. They require a special bonding technique to be attached to the corresponding position, which is a prominent disadvantage for our design where a large number of diodes are used.

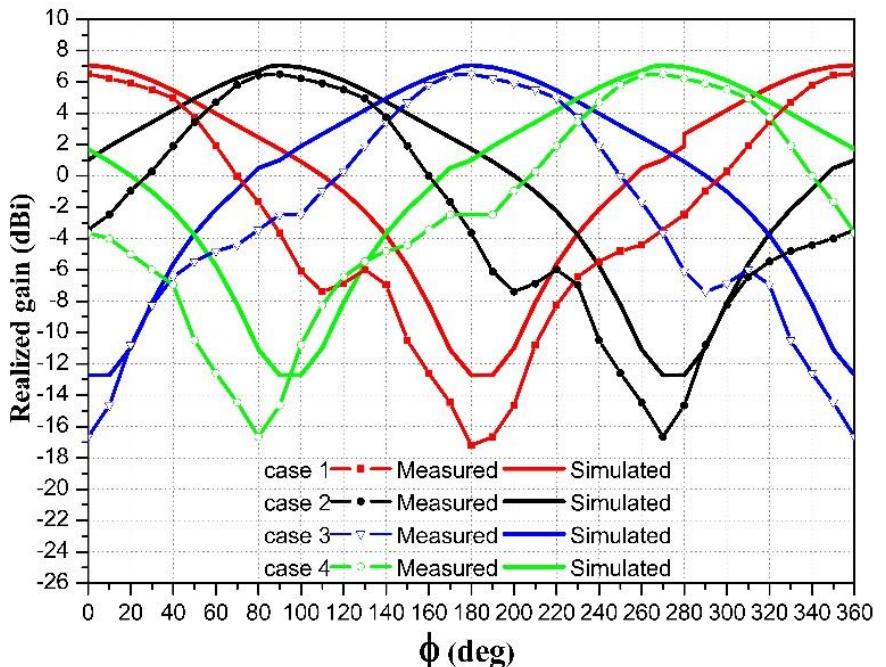

(a)

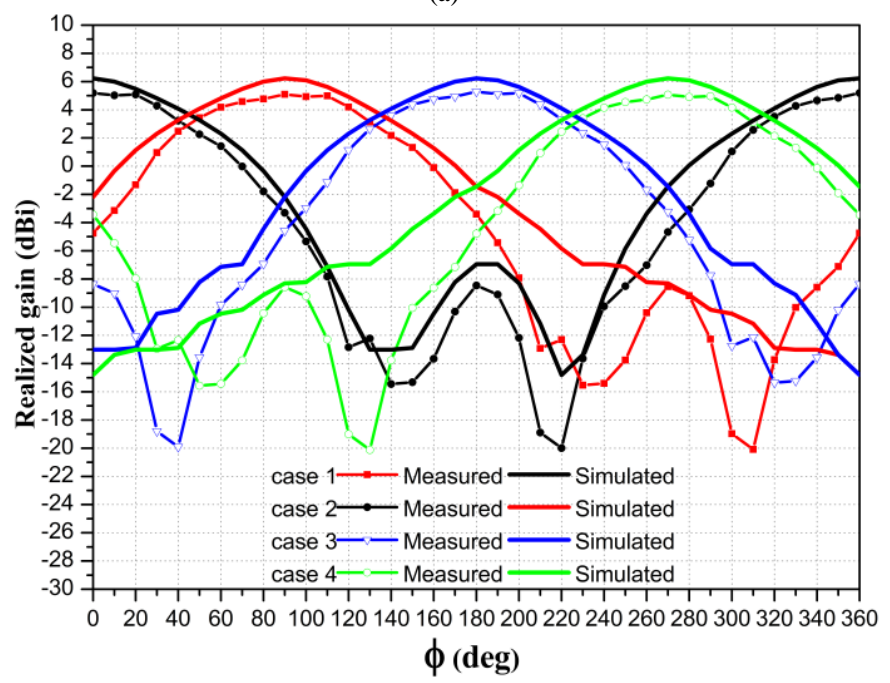

(b)

Fig. 12. Measured gains of the switching beams at (a) $2.5 \mathrm{GHz}$; (b) $5.3 \mathrm{GHz}$.

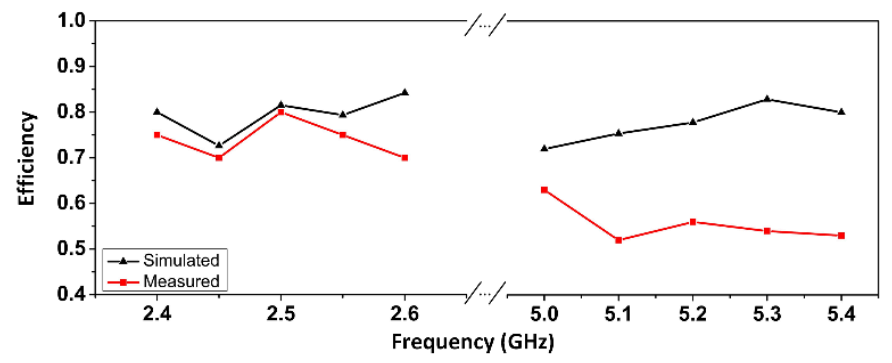

Fig. 13. Simulated and measured efficiency at two frequency bands.

The resulting antenna has dimensions of approximately 54 $\mathrm{mm} \times 54 \mathrm{~mm} \times 204 \mathrm{~mm}$. In addition, it is light in weight. A comparison between the dual-band AFSS antenna in our work and the cited single-band antennas is given in Table III. It is clear that the size of the presented antenna is smaller than any of the antennas in the literature.

TABLE III

COMPARISON BETWEEN THIS WORK AND REPORTED WORK

\begin{tabular}{cccc}
\hline Ref.NO. & Operation mode & Gain & Dimensions $(\mathrm{mm})$ \\
\hline$[17]$ & Single band & $8.2 \mathrm{dBi}$ & $92 \times 92 \times 220$
\end{tabular}


Our work

$\begin{array}{lcc}\text { Single band } & 13 \mathrm{dBi} & 100 \times 100 \times 565 \\ \text { Single band } & 10 \mathrm{dBi} & 150 \times 150 \times 190 \\ \text { Single band } & 6.6-7 \mathrm{dBi} & 100 \times 100 \times 278 \\ \text { Single band } & 7 \mathrm{dBi} & 92 \times 92 \times 150 \\ \text { Single band } & 8.7 \mathrm{dBi} & 216 \times 216 \times 200\end{array}$

dual band $\quad 5-6.5 d B i \quad 54 \times 54 \times 204$

\section{CONCLUSION}

Dual-band electronic beam-switching can be achieved using the cylindrical AFSS antenna described in this paper. AFSS consisting of slot-loop unit cells are suitable for this application. A double-sided biasing technique has been employed to reconfigure the AFSS between reflective and transparent states. By switching on the PIN diodes in one sector of the AFSS, the omnidirectional patterns of the dual-band feed dipole can be transformed to directive beams. To predict and validate the AFSS antenna performance, a dual-band metallic semicylindrical reflector antenna was simulated and fabricated as a reference antenna. The measured results of the AFSS antenna compared well with the reference antenna. The measured reflection coefficients are acceptable at the two operating frequency ranges. It is shown that the realized $\mathrm{H}$-plane gains of the proposed antenna are $6.5 \mathrm{dBi}$ and $5 \mathrm{dBi}$, respectively. Furthermore, it can be proved that the antenna possesses the capability of switching the beams to cover the entire horizontal plane in 4 steps. With its compact size, light weight, and low cost, the proposed antenna can be a candidate to be used in indoor wireless or base-station communication systems.

\section{REFERENCES}

[1] J. W. Lu, D. Ireland, and R. Schlub, "Dielectric embedded ESPAR (DEESPAR) antenna array for wireless communications," IEEE Trans. Antennas Propag., vol. 53, no. 8, pp. 2437-2443, Aug, 2005.

[2] W. H. Chen, J. W. Sun, X. Wang, Z. H. Feng, F. L. Chen, Y. Furuya, and A. Kuramoto, "A novel planar switched parasitic array antenna with steered conical pattern," IEEE Trans. Antennas Propag., vol. 55, no. 6, pp. 1883-1887, Jun, 2007.

[3] M. I. Lai, T. Y. Wu, J. C. Hsieh, C. H. Wang, and S. K. Jeng, "Compact switched-beam antenna employing a four-element slot antenna array for digital home applications," IEEE Trans. Antennas Propag., vol. 56, no. 9, pp. 2929-2936, Sep, 2008.

[4] J. R. De Luis, and F. De Flaviis, "Frequency agile switched beam antenna array system," IEEE Trans. Antennas Propag., vol. 58, no. 10, pp. 31963204, 2010.

[5] M. N. Jazi, and T. A. Denidni, "Agile Radiation-Pattern Antenna Based on Active Cylindrical Frequency Selective Surfaces," IEEE Antennas Wireless Propag. Lett., vol. 9, pp. 387-388, 2010.

[6] H. T. Liu, S. Gao, and T. H. Loh, "Electrically Small and Low Cost Smart Antenna for Wireless Communication," IEEE Trans. Antennas Propag., vol. 60, no. 3, pp. 1540-1549, Mar, 2012.

[7] H. T. Liu, S. Gao, and T. H. Loh, "Small Director Array for Low-Profile Smart Antennas Achieving Higher Gain," IEEE Trans. Antennas Propag., vol. 61, no. 1, pp. 162-168, Jan, 2013.

[8] P. Y. Qin, Y. J. Guo, and C. Ding, “A Beam Switching Quasi-Yagi Dipole Antenna," IEEE Trans. Antennas Propag., vol. 61, no. 10, pp. 4891-4899, Oct, 2013.
[9] R. Guzman-Quiros, A. R. Weily, J. L. Gomez-Tornero, and Y. J. Guo, “A Fabry-Pérot Antenna With Two-Dimensional Electronic Beam Scanning," IEEE Trans. Antennas Propag., vol. 64, no. 4, pp. 1536-1541, Apr, 2016.

[10] X. S. Yang, B. Z. Wang, W. X. Wu, and S. Q. Xiao, "Yagi patch antenna with dual-band and pattern reconfigurable characteristics," IEEE Antennas Wireless Propag. Lett., vol. 6, pp. 168-171, 2007.

[11] M. Maqsood, S. Gao, T. W. C. Brown, M. Unwin, R. D. Van Steenwijk, J. D. Xu, and C. I. Underwood, "Low-Cost Dual-Band Circularly Polarized Switched-Beam Array for Global Navigation Satellite System," IEEE Trans. Antennas Propag., vol. 62, no. 4, pp. 1975-1982, Apr, 2014.

[12] A. Pal, A. Mehta, D. Mirshekar-Syahkal, P. Deo, and H. Nakano, "DualBand Low-Profile Capacitively Coupled Beam-Steerable Square-Loop Antenna," IEEE Trans. Antennas Propag., vol. 62, no. 3, pp. 1204-1211, Mar, 2014.

[13] H. Boutayeb, T. Brillat, J. Daniel, F. Gadot, P. Garel, A. De Lustrac, K. Mahdjoubi, P. Ratajczak, and A.-C. Tarot, " A reconfigurable electromagnetic bandgap structure for a beam steering base station antenna," in Workshop on Innovative Periodic Antennas, Santiago de Compostela, Spain, pp. 383-389, 2004.

[14] H. Boutayeb, T. A. Denidni, K. Mahdjoubi, A. C. Tarot, A. R. Sebak, and L. Talbi, "Analysis and design of a cylindrical EBG-based directive antenna," IEEE Trans. Antennas Propag., vol. 54, no. 1, pp. 211-219, Jan, 2006.

[15] A. Edalati, and T. A. Denidni, "Reconfigurable Beamwidth Antenna Based on Active Partially Reflective Surfaces," IEEE Antennas Wireless Propag. Lett., vol. 8, pp. 1087-1090, 2009.

[16] M. N. Jazi, and T. A. Denidni, "Agile Radiation-Pattern Antenna Based on Active Cylindrical Frequency Selective Surfaces," IEEE Antennas Wireless Propag. Lett., vol. 9, pp. 387-388, 2010.

[17] M. N. Jazi, and T. A. Denidni, "Frequency Selective Surfaces and Their Applications for Nimble-Radiation Pattern Antennas," IEEE Trans. Antennas Propag., vol. 58, no. 7, pp. 2227-2237, Jul, 2010.

[18] A. Edalati, and T. A. Denidni, "High-Gain Reconfigurable Sectoral Antenna Using an Active Cylindrical FSS Structure," IEEE Trans. Antennas Propag., vol. 59, no. 7, pp. 2464-2472, Jul, 2011.

[19] A. Edalati, and T. A. Denidni, "Frequency Selective Surfaces for BeamSwitching Applications," IEEE Trans. Antennas Propag., vol. 61, no. 1, pp. 195-200, Jan, 2013.

[20] L. Zhang, Q. Wu, and T. A. Denidni, "Electronically Radiation Pattern Steerable Antennas Using Active Frequency Selective Surfaces," IEEE Trans. Antennas Propag., vol. 61, no. 12, pp. 6000-6007, Dec, 2013.

[21] M. Niroo-Jazi, and T. A. Denidni, "Electronically Sweeping-Beam Antenna Using a New Cylindrical Frequency-Selective Surface," IEEE Trans. Antennas Propag., vol. 61, no. 2, pp. 666-676, Feb, 2013.

[22] W. B. Pan, C. Huang, P. Chen, M. B. Pu, X. L. Ma, and X. G. Luo, "A Beam Steering Horn Antenna Using Active Frequency Selective Surface," IEEE Trans. Antennas Propag., vol. 61, no. 12, pp. 6218-6223, Dec, 2013.

[23] B. Liang, B. Sanz-Izquierdo, E. A. Parker, and J. C. Batchelor, "Cylindrical Slot FSS Configuration for Beam-Switching Applications," IEEE Trans. Antennas Propag., vol. 63, no. 1, pp. 166-173, Jan, 2015.

[24] B. Sanz-Izquierdo, E. A. Parker, J. B. Robertson, and J. C. Batchelor, "Tuning technique for active FSS arrays," Electron. Lett., vol. 45, no. 22, pp. 1107-1108, Oct 22, 2009.

[25] B. Sanz-Izquierdo, E. A. Parker, and J. C. Batchelor, "Dual-Band Tunable Screen Using Complementary Split Ring Resonators," IEEE Trans. Antennas Propag., vol. 58, no. 11, pp. 3761-3765, Nov, 2010. 